\title{
THE TWO-TYPE CONTINUUM RICHARDSON MODEL: NONDEPENDENCE OF THE SURVIVAL OF BOTH TYPES ON THE INITIAL CONFIGURATION
}

\author{
SEBASTIAN CARSTENS, ${ }^{*}$ Ludwig-Maximilians-Universität Müchen \\ THOMAS RICHTHAMMER, ${ }^{* * *}$ University of California, Los Angeles
}

\begin{abstract}
We consider the model of Deijfen, Häggström and Bagley (2004) for competing growth of two infection types in $\mathbb{R}^{d}$, based on the Richardson model on $\mathbb{Z}^{d}$. Stochastic ball-shaped infection outbursts transmit the infection type of the center to all points of the ball that are not yet infected. Relevant parameters of the model are the initial infection configuration, the (type-dependent) growth rates, and the radius distribution of the infection outbursts. The main question is that of coexistence: Which values of the parameters allow the unbounded growth of both types with positive probability? Deijfen, Häggström and Bagley (2004) conjectured that the initial configuration is basically irrelevant for this question, and gave a proof for this under strong assumptions on the radius distribution, which, e.g. do not include the case of a deterministic radius. Here we give a proof that does not rely on these assumptions. One of the tools to be used is a slight generalization of the model with immune regions and delayed initial infection configurations.
\end{abstract}

Keywords: Continuum growth model; Richardson's model; competing growth; initial configuration; shape theorem

2010 Mathematics Subject Classification: Primary 60K35

Secondary 82B 43

\section{Introduction}

We consider a model for the competing growth of $n$ types of infection on $\mathbb{R}^{d}$, where $d \geq 1$ is the number of spatial dimensions. We will refer to it as the ( $d$-dimensional $n$-type) continuum Richardson model (CR model). The corresponding model with one infection type was introduced by Deijfen [1] as a continuum version of the growth model on $\mathbb{Z}^{d}$ introduced by Richardson [6]. The multitype versions of these models were first considered by Häggström and Pemantle [5] (discrete model) and Deijfen et al. [4] (continuum model).

The CR model is a stochastic process $Z=\left(Z_{t}^{i}\right)_{i, t}$, where $Z_{t}^{i}$ denotes the subset of $\mathbb{R}^{d}$ that is infected with type $i \in\{1, \ldots, n\}$ (i.e. $i$-infected) at time $t \geq 0$. Initially, given disjoint regions $\Gamma_{i}$ are $i$-infected. Whenever a region is $i$-infected, it stays like that. Furthermore, it tries to $i$-infect healthy 'neighboring' regions by means of stochastic infection outbursts. The waiting time for the first outburst of infection type $i$ after time $t$ is exponentially distributed with rate $\beta_{i} \lambda^{d}\left(Z_{t}^{i}\right)$, where $\lambda^{d}$ denotes the Lebesgue measure and $\beta_{i}$ is a type-dependent growth rate. The outburst has the shape of a ball, where the center is chosen uniformly in $Z_{t}^{i}$ and the radius

Received 17 August 2009; revision received 8 September 2010.

* Postal address: Mathematisches Institut, Ludwig-Maximilians-Universität Müchen, Theresienstr. 39, D-80333

München, Germany.

** Email address: richth@math.lmu.de

Research supported in part by NSF grant DMS-0300672. 
is chosen with respect to a given radius distribution $\rho$. At the time of the outburst all points within the ball that are not yet infected become $i$-infected. We note that the $i$-infected region $Z_{t}^{i}$ is increasing in $t$, and at any given time $t$ the sets $\left(Z_{t}^{i}\right)_{i}$ are disjoint.

The parameters of the CR model are the initial configuration $\Gamma=\left(\Gamma_{1}, \ldots, \Gamma_{n}\right)$, the growth rates $\beta=\left(\beta_{1}, \ldots, \beta_{n}\right)$, and the radius distribution $\rho$. Throughout the paper, we will assume that $\lambda^{d}\left(\Gamma_{i}\right)>0, \beta_{i}>0$, and $\rho(\{0\})=0$ to avoid trivialities; furthermore, we will assume that $\Gamma_{i}$ is bounded and that $\rho$ satisfies

$$
\int_{0}^{\infty} r^{d} \mathrm{~d} \rho(r)<\infty,
$$

which will ensure that the model is well defined. Other assumptions on $\rho$ that sometimes play an important role are stronger moment assumptions such as

$$
\int_{0}^{\infty} \mathrm{e}^{\varphi r} \mathrm{~d} \rho(r)<\infty \quad \text { for some } \varphi>0,
$$

and conditions on the support of $\rho$ such as

$$
\rho((0, \delta))>0 \text { for all } \delta>0 .
$$

For instance, in [1] and [4] the authors showed, for the one-type CR model, that, under condition (2) on $\rho$, the asymptotic shape of $Z_{t} / t$ for $t \rightarrow \infty$ is a ball; we have stated this result as Theorem 3 in Section 2.3.

An interesting question for the CR model is that of coexistence: Do all infection types grow unboundedly at the same time with positive probability? In the two-type CR model it is conjectured that we have coexistence if and only if $\beta_{1}=\beta_{2}$. It is known that, for fixed $\beta_{1}$, at most countably many values of $\beta_{2}$ allow coexistence (see [4]) and the value $\beta_{2}=\beta_{1}$ is one of them (see [2]). Both results assume condition (2) and concern models with special initial configurations consisting of two disjoint balls. However, the question of coexistence is basically independent of the initial configuration. This is shown in [4], assuming that $\rho$ satisfies (2) and (3). The aim of the present paper is to show how these extra assumptions can be avoided. (In fact, it turns out that assuming condition (1) is sufficient.) As an immediate consequence, the aforementioned coexistence results extend to basically all initial configurations for all radius distributions satisfying (2). This is desirable as some of the most natural choices of $\rho$ do not satisfy (3), e.g. the case of outbursts with a deterministic radius.

For stating our result, let us consider a two-type CR model with initial condition $\Gamma=\left(\Gamma_{1}, \Gamma_{2}\right)$ and growth rates $\beta=\left(\beta_{1}, \beta_{2}\right)$. Let $B_{\Gamma}$ denote the smallest ball centered at the origin containing $\Gamma_{\cup}:=\Gamma_{1} \cup \Gamma_{2}$. Let $L_{i}$ be the event that type $i$ leaves $B_{\Gamma}$, let $G_{i}$ be the event that type $i$ reaches points arbitrarily far from the origin, and let $G:=G_{1} \cap G_{2}$ be the event of unbounded growth of both types. Whenever we consider more than one model, we will indicate the relevant parameters in parentheses after the corresponding event.

Theorem 1. We consider two $d$-dimensional two-type $C R$ models with $d \geq 2$, initial configuration $\Gamma$ and $\Gamma^{\prime}$, growth rates $\beta_{i}=\beta_{i}^{\prime}$, and radius distributions $\rho=\rho^{\prime}$ (satisfying (1)). If we have $\mathrm{P}\left(L_{i}(\Gamma)\right)>0$ and $\mathrm{P}\left(L_{i}\left(\Gamma^{\prime}\right)\right)>0$ for $i=1,2$, the possibility of coexistence does not depend on the initial condition:

$$
\mathrm{P}(G(\Gamma))>0 \quad \Longleftrightarrow \quad \mathrm{P}\left(G\left(\Gamma^{\prime}\right)\right)>0 .
$$


We note that the condition on the events $L_{i}$ merely states that in the initial configurations no type strangles the other. As mentioned above, combining Theorem 1 with the coexistence results from [2] and [4] immediately gives the following result.

Theorem 2. We consider a d-dimensional two-type CR model with $d \geq 2$, initial configuration $\Gamma$, growth rates $\beta=\left(\beta_{1}, \beta_{2}\right)$, and radius distribution $\rho$ satisfying (2).

(a) If $\beta_{1}=\beta_{2}>0$ and $\mathrm{P}\left(L_{i}(\beta)\right)>0$ for $i=1,2$, we have $\mathrm{P}(G(\beta))>0$.

(b) For fixed $\beta_{1}$, we have $\mathrm{P}(G(\beta))=0$ for all but countably many values of $\beta_{2}$.

We briefly discuss to what extent the proof of Theorem 1 of [4] makes use of the stronger assumptions on the radius distribution. One of the tools used is the aforementioned theorem on the asymptotic shape of the infected region, which relies on (2). But only the lower bound of the asymptotic shape is used, which is still valid if the radii of the outbursts are increased. Thus, it is easy to relax (2) to (1). Another tool in the proof is the construction of an infection evolution that infects some given points with type 1 ('type-1 points') and some other given points with type 2 ('type-2 points'). For this, condition (3) is essential. Suppose that some type-1 points are surrounded by type-2 points. Utilizing (3) it is easy to construct a sequence of sufficiently small outbursts that 1 -infect the type-1 points without 1 -infecting the type-2 points. Without (3), this seems to be hopeless. Instead, our strategy will be to 2-infect all type-2 points, but to 1-infect only a single (suitably chosen) type-1 point. The construction of such an infection evolution turns out to be possible without (3), though somewhat complicated in terms of geometry. We then have to investigate how the unknown infection states of the remaining type-1 points affect the subsequent infection evolution. Our key tool for this is a generalized version of the CR model that allows immune regions and delayed infections in its initial configuration.

We define a generalized initial configuration $\Gamma$ of an $n$-type CR model to be a finite collection $\Gamma=\left(\Gamma_{j}\right)$ of disjoint bounded Borel subsets of $\mathbb{R}^{d}$, each of which has an associated type $i\left(\Gamma_{j}\right) \in\{1, \ldots, n\}$ and an associated time $t\left(\Gamma_{j}\right) \in[0, \infty]$. We consider $\Gamma_{j}$ to be uninfected at times $t<t\left(\Gamma_{j}\right)$ and infected with type $i\left(\Gamma_{j}\right)$ at times $t \geq t\left(\Gamma_{j}\right)$. If $t\left(\Gamma_{j}\right)<\infty$, this corresponds to a delayed initial configuration, and if $t\left(\Gamma_{j}\right)=\infty$, this corresponds to an immune region (and in this case $i\left(\Gamma_{j}\right)$ is irrelevant). We will use the shorthand notation $\Gamma_{\cup}:=\bigcup_{j} \Gamma_{j}$. Additionally, we are given growth parameters $\beta_{i}>0$ and a radius distribution $\rho$ as before. The corresponding stochastic process will again be denoted by $Z=\left(Z_{t}^{i}\right)_{i, t}$, where $Z_{t}^{i}$ denotes the $i$-infected subset of $\mathbb{R}^{d}$ at time $t \geq 0$. The dynamics of outbursts is a generalization of that for the CR model with standard initial configurations. It is defined by the following properties.

- $Z$ is a Markov process (which is time homogeneous if and only if $t\left(\Gamma_{j}\right) \in\{0, \infty\}$ for all $j$ ).

- In $\Gamma_{\cup}$ we have a deterministic infection evolution as described above.

- Regions of $\Gamma_{\cup}^{\mathrm{c}}$ can only be infected by ball-shaped infection outbursts. If the center of such a ball is $i$-infected then the outburst $i$-infects all points within the ball that are not yet infected and not in $\Gamma_{\cup}$.

- At a given time $t$ an outburst of type $i$ occurs at rate $\beta_{i} \lambda^{d}\left(Z_{t}^{i}\right)$. The center of the corresponding ball is chosen uniformly in $Z_{t}^{i}$ and the radius is chosen with respect to $\rho$.

The above properties uniquely define the distribution of the process $Z$. We will refer to such a process as the $\mathrm{CR}$ model with generalized initial configuration or the generalized CR model. 
This paper is organized as follows. In Section 2 we clarify some notation, describe two ways to construct a generalized CR model from a Poisson point process, and state a shape theorem and some other important properties of the generalized CR model. The proofs of the corresponding lemmas and theorems are relegated to Section 3. In Section 4 we prove Theorem 1. The proofs of the corresponding lemmas are relegated to Section 5 .

\section{Setting}

\subsection{The underlying space and point process}

First we introduce some notation concerning subsets of $\mathbb{R}^{d}$ and point processes. We denote the Lebesgue measure on $\mathbb{R}^{d}$ by $\lambda^{d}$ and the restriction of $\lambda=\lambda^{1}$ to $\mathbb{R}_{+}=[0, \infty)$ by $\lambda_{+}$. Let $B(x, r)$ denote the closed ball with center $x \in \mathbb{R}^{d}$ and radius $r>0$. Let $d(x, y):=|x-y|$ be the Euclidean distance between two points $x, y \in \mathbb{R}^{d}$. We will use $d(\cdot, \cdot)$ in the usual way also for the distance between a point and a set or the distance between two sets. For $A \subset \mathbb{R}^{d}$ and $r>0$, let

$$
A_{+r}=\left\{x \in \mathbb{R}^{d}: d(x, A) \leq r\right\}
$$

Considering point processes, we will be mainly concerned with points $p=(x, s, r, w) \in$ $\mathbb{R}^{d} \times \mathbb{R}_{+} \times \mathbb{R}_{+} \times \mathbb{R}_{+}=: \mathbb{R}_{\times}^{d}$. We will refer to the components of such a point $p$ as position $x$, time $s$, radius $r$, and strength $w$. For $A \subset \mathbb{R}^{d}$ and time interval $I \subset \mathbb{R}_{+}$, by abuse of notation we will sometimes consider $A$ and $A \times I$ as subsets of $\mathbb{R}_{\times}^{d}$. A point configuration $X$ is defined as a locally finite subset of $\mathbb{R}_{\times}^{d}$. Let $\mathcal{X}$ denote the set of all point configurations. For a Borel set $E \subset \mathbb{R}_{\times}^{d}$ and $X \in \mathcal{X}$, let $N_{E}(X)$ be the number of points of $X$ in $E$, and let $\mathcal{F}_{E}$ be the $\sigma$-algebra on $\mathcal{X}$ generated by all counting variables $N_{E^{\prime}}$, where $E^{\prime}$ is a Borel subset of $E$. We define $\mathcal{F}:=\mathcal{F}_{\mathbb{R}_{x}^{d}}$ to be the $\sigma$-algebra usually associated to $\mathcal{X}$. Sets $M \in \mathcal{F}_{E}$ will be called events, depending only on (the information in) $E$. For a given radius distribution $\rho$, we consider the Poisson point process with intensity measure $\lambda_{x}^{d}:=\lambda^{d} \otimes \lambda_{+} \otimes \rho \otimes \lambda_{+}$and denote its distribution by $\mathrm{P}$.

\subsection{Constructing the model from a Poisson point process}

Many proofs rely on the comparison of different generalized CR models, so we have to construct suitable couplings between them. To prepare this, we describe two methods to construct an infection evolution $Z=Z(X)$ for a given point configuration $X \in X$. Typically, $X$ will be a realization of $P$.

The first method to construct an infection evolution from $X$ is called 'scanning from the time of infection'. The infection evolution in the generalized initial configuration $\Gamma \cup$ is deterministic: every region $\Gamma_{j}$ is infected at the associated time with the associated type (and is uninfected before). Whenever a previously uninfected region $A$ is $i$-infected at some time $t$, it gets a scanning device that starts scanning for points of $X$ in $A \times[t, \infty) \times \mathbb{R}_{+} \times\left[0, \beta_{i}\right]$. A point $p=(x, s, r, w) \in X$ located in $A$ with time $s \geq t$ and strength $w \leq \beta_{i}$ will be scanned at time $s$. At this time the point $p$ produces an outburst $i$-infecting all points of $B(x, r)$ that are not yet infected and not in $\Gamma_{\cup}$. We stress that in this construction a point $p=(x, s, r, w) \in X$ can produce an outburst only if $x$ has been infected by time $s$; if $x$ has been $i$-infected before time $s$ and $w \leq \beta_{i}, p$ produces an outburst at time $s$.

This construction is changed slightly in the second method: 'scanning from time 0'. The infection evolution in $\Gamma_{\cup}$ is the same as above, and again a previously uninfected region $A$ that is $i$-infected at some time $t$ gets a scanning device. But now this device starts scanning for points of $X$ in $A \times[0, \infty) \times \mathbb{R}_{+} \times\left[0, \beta_{i}\right]$. So a point $p=(x, s, r, w) \in X$ located in $A$ with 
time $s \geq 0$ and strength $w \leq \beta_{i}$ will be scanned at time $t+s$. The outburst generated by $p$ at this time has the same effect as above. We stress that in this construction the only condition for a point $p=(x, s, r, w) \in X$ to produce an outburst is that $w$ is sufficiently small (depending on the eventual infection type of $x$ ); if $x$ gets $i$-infected at time $t$ and $w \leq \beta_{i}, p$ produces an outburst at time $t+s$.

If $X$ is chosen according to $\mathrm{P}$, both constructions give a random infection evolution with the properties described in Section 1, and thus yield a generalized CR model. Furthermore, for both methods of construction, there are only finitely many outbursts in any finite amount of time almost surely (a.s.). This can be shown as in [1, Proposition 2.1] for the usual one-type CR model, making use of property (1) of the radius distribution $\rho$. As a consequence of the properties of the Poisson point process, in both methods of construction, a.s., no point is scanned at the same time as another point or at a time associated to part of the initial configuration. The only instance where we will use 'scanning from time 0 ' is in the proof of Theorem 4. In every other instance, when a CR model is related to a point process or a point configuration, we will assume that the CR model is constructed by 'scanning from the time of infection'. Whenever we consider two or more initial configurations, we write $Z_{t}^{i}(\Gamma)$ to indicate which initial configuration the process uses. In the special case of a standard initial configuration, the above constructions are slightly easier; see [1], [2], and [4].

We conclude this subsection by introducing some notions useful for describing an infection evolution. The total infected region at time $t$ will be denoted by $Z_{t}^{\cup}:=Z_{t}^{1} \cup \cdots \cup Z_{t}^{n}$. Next we consider a point $p=(x, s, r, w)$ scanned at time $t$. The point $p$ is said to produce an effective outburst if it produces an outburst that increases the infected region, i.e. $B(x, r) \not \subset Z_{t}^{\cup} \cup \Gamma \cup$. The point $p$ is said to produce a virtual effective outburst if $w \leq \max _{i} \beta_{i}$ and $B(x, r) \not \subset Z_{t}^{\cup} \cup \Gamma \cup$ (but $x$ is not necessarily infected at time $s$, so $p$ might not produce any outburst). A virtual effective outburst is said to virtually infect points of a set $C$ if $B(x, r) \cap C \not \subset Z_{t}^{\cup} \cup \Gamma \cup$. A time $t$ is called a time of growth if at this time the infected region increases, which may be due to the initial configuration or an effective outburst. Many proofs of assertions about an infection evolution are by induction on the times of growth (considered in linear order). Finally, we call a sequence $\left(p_{n}\right)_{n}$ of points $p_{n}$ an $i$-infection path if every $p_{n}$ produces an effective outburst that $i$-infects the position of $p_{n+1}$.

\subsection{Auxiliary results}

Let $Z$ denote a two-type $C R$ model with standard initial configuration $\Gamma=\left(\Gamma_{1}, \Gamma_{2}\right)$. In the proof of Theorem 1 we have to construct various infection evolutions. A building block of these constructions is to let one type, say type 1 , grow along a given set $C$. If at time $T_{1}$ a neighborhood of some point $c_{0} \in C$ is 1 -infected and $C$ is not 2-infected, we construct an infection evolution in the time interval $\left(T_{1}, T_{2}\right]$ so that type 2 does not grow at all and type 1 infects some enlargement of $C$ (as far as that is possible). For this, we use outbursts of a certain size, so we choose a $d_{0}>0$ with

$$
\rho\left(\left[d_{0}, d_{0}+\varepsilon\right]\right)>0 \text { for all } \varepsilon>0,
$$

e.g. $d_{0}:=\inf \left\{r>r_{0}: \rho([0, r])>\rho\left(\left[0, r_{0}\right]\right)\right\}$ for some small $r_{0}$ with $\rho\left(\left[0, r_{0}\right]\right)<1$. We fix the value of such a $d_{0}$ for the rest of the paper. Definition 1 below gives a more precise description of the growth of type 1 along a set $C$, and in Lemma 1 below we give conditions on $C$ under which this kind of growth is possible.

Definition 1. (Growth along a set.) Let $0<\delta<d_{0} / 2,0 \leq T_{1}<T_{2}, B, C \subset \mathbb{R}^{d}$ be bounded Borel sets with $C_{+d_{0}+2 \delta} \subset B$, and $c_{0} \in C$. An event $M$ of point configurations is said to 
describe the growth of type 1 along $C$ (within $B$, starting at $\left.c_{0}\right)$ in the time interval $\left(T_{1}, T_{2}\right.$ ] using outbursts of size $d_{0}$ with precision $\delta$ if it has the following properties: $M$ depends only on $B \times\left(T_{1}, T_{2}\right], \mathrm{P}(M)>0$, and

$$
\begin{aligned}
& \left\{B\left(c_{0}, \delta\right) \subset Z_{T_{1}}^{1}, Z_{T_{1}}^{\cup} \subset B, C_{+\delta} \cap Z_{T_{1}}^{2}=\varnothing\right\} \cap M \\
& \quad \subset\left\{C_{+d_{0}-2 \delta}-Z_{T_{1}}^{2} \subset Z_{T_{2}}^{1} \subset C_{+d_{0}+2 \delta} \cup Z_{T_{1}}^{1}, Z_{T_{2}}^{2}=Z_{T_{1}}^{2}\right\} .
\end{aligned}
$$

Lemma 1. (Constructing infection evolutions.) In the situation of Definition 1 suppose that the set $C$ is of one of the following types.

(a) $C=\left\{c_{0}, \ldots, c_{n}\right\}$, where $d\left(\left\{c_{0}, \ldots, c_{i-1}\right\}, c_{i}\right) \leq d_{0}-2 \delta$ for all $i \geq 1$.

(b) $C$ is a bounded, pathwise connected set.

Then it is possible to let type 1 grow along $C$ (within $B$, starting at $c_{0}$ ) in the time interval $\left(T_{1}, T_{2}\right]$ using outbursts of size $d_{0}$ with precision $\delta$.

We note that Lemma 1 and Definition 1 apply analogously when the roles of types 1 and 2 are interchanged. An important special case of Lemma 1(b) is that $C$ is the trace of some continuous curve $\gamma:[0,1] \rightarrow \mathbb{R}^{d}$ with $c_{0}=\gamma(0)$.

For a given CR model and a Borel set $B$, let $\tau_{B}$ denote the first time all points of $B$ are infected, and let $\zeta_{B}$ denote the time of the last effective outburst produced by a point with position in $B$. These random variables have finite values.

Lemma 2. Let $Z$ be a d-dimensional n-type $C R$ model with standard initial configuration, and let $B \subset \mathbb{R}^{d}$ be a bounded Borel set. We have, a.s.,

(a) $\tau_{B}<\infty$, i.e. $B$ is completely infected after a finite time,

(b) $\zeta_{B}<\infty$, i.e. only finitely many effective outbursts originate from $B$.

Very similar results have been obtained before. Note that in Lemma 2(b) we do not assume condition (2) on the radius distribution (compare to Lemma 4.5(a) of [4]). The proof of part (b) basically relies on the shape theorem stated below. For its proof, we refer the reader to [4].

Theorem 3. Let $Z$ be a d-dimensional one-type $C R$ model with standard initial configuration $\Gamma$, growth rate $\beta$, and radius distribution $\rho$ satisfying (2). There exists a $\mu>0$ (independent of $\beta$ and $\Gamma$ ) such that, for all $0<\varepsilon<1$, we have, a.s.,

$$
(1-\varepsilon) B\left(0, \frac{\beta}{\mu}\right) \subset \frac{Z_{t}}{t} \subset(1+\varepsilon) B\left(0, \frac{\beta}{\mu}\right) \text { for sufficiently large } t .
$$

We need a version of Lemma 2 for a CR model $\tilde{Z}$ with generalized initial configuration $\tilde{\Gamma}$. There may be regions that never become infected since they are enclosed by a thick layer of immune region. So we consider $B_{\tilde{\Gamma}}$, the smallest ball enclosing $\tilde{\Gamma}_{\cup}$ centered at the origin, and $L$, the event that the infection leaves $B_{\tilde{\Gamma}}$. Let $\tilde{\zeta}_{B}$ be the time of the last virtual outburst that is produced by a point with position in $B$ and virtually infecting points outside $B_{\tilde{\Gamma}}$.

Lemma 3. Let $\tilde{Z}$ be a d-dimensional n-type CR model with generalized initial configuration $\tilde{\Gamma}$, and let $B \subset \mathbb{R}^{d}$ be a bounded Borel set. On $L$ we have, a.s.,

(a) $\tau_{B-B_{\tilde{\Gamma}}}<\infty$, i.e. $B-B_{\tilde{\Gamma}}$ is completely infected after a finite time, 
(b) $\tilde{\zeta}_{B}<\infty$, i.e. there are only finitely many virtual effective outbursts originating from $B$ virtually infecting points outside $B_{\tilde{\Gamma}}$.

Again, part (b) of Lemma 3 relies on a corresponding version of the shape theorem.

Theorem 4. Let $\tilde{Z}$ be a d-dimensional one-type CR model with generalized initial configuration $\tilde{\Gamma}$, growth rate $\beta$, and radius distribution $\rho$ satisfying (2). There exists a $\mu>0$ (independent of $\beta, \tilde{\Gamma})$ such that, for all $0<\varepsilon<1$, we have, a.s., on $L$,

$$
(1-\varepsilon) B\left(0, \frac{\beta}{\mu}\right) \subset \frac{\tilde{Z}_{t} \cup B_{\tilde{\Gamma}}}{t} \subset(1+\varepsilon) B\left(0, \frac{\beta}{\mu}\right) \text { for sufficiently large } t .
$$

\section{Proofs of auxiliary results}

For the following proofs, we set $\beta_{\wedge}:=\min _{i} \beta_{i}$ and $\beta_{\vee}:=\max _{i} \beta_{i}$.

\subsection{Constructing infection evolutions: Lemma 1}

In both cases (a) and (b) we define an event $M$ that can be easily checked to induce the desired infection evolution. For (a), let

$$
t_{i}:=T_{1}+\frac{i}{n+1}\left(T_{2}-T_{1}\right) \quad(0 \leq i \leq n+1) \quad \text { and } \quad B_{i}:=B\left(c_{i}, \delta\right) \quad(0 \leq i \leq n) .
$$

Let $M$ be the set of all point configurations with exactly one point in each of $B_{i} \times\left(t_{i}, t_{i+1}\right] \times$ $\left[d_{0}, d_{0}+\delta\right) \times\left[0, \beta_{1}\right]$ for all $0 \leq i \leq n$, and no other points apart from these in $B \times\left(T_{1}, T_{2}\right] \times$ $\left[0, \beta_{\vee}\right]$.

For (b), we note that the compact set $C_{+d_{0}-2 \delta}$ is covered by the union of all open balls with center in $C$ and radius $d_{0}-\delta$. We choose a finite subcover consisting of balls with centers $c_{1}, \ldots, c_{n}$. Without loss of generality we assume that $d\left(c_{i-1}, c_{i}\right) \leq d_{0}-2 \delta$ for $1 \leq i \leq n$ (by joining the points $c_{i}$ by continuous curves within $C$ and adding points of these curves that are sufficiently close to each other to this list of centers). Let $M$ be the event constructed in (a) for this choice of points $\left\{c_{0}, \ldots, c_{n}\right\}$.

\subsection{Standard initial configuration: Lemma 2}

For the proof of part (a), we assume that $B$ is a ball with $B \supset \Gamma$ (by replacing $B$ with a sufficiently big ball). Let $\delta<d_{0} / 2$. As in the proof of Lemma 1(b), we can choose $c_{1}, \ldots, c_{n} \in B$ such that $d\left(c_{i-1}, c_{i}\right) \leq d_{0}-2 \delta$ and $\bigcup_{i} B\left(c_{i}, d_{0}-\delta\right) \supset B$. We set $C_{0}=\Gamma$ and $C_{i}=B\left(c_{i}, \delta\right)$, and, for given $T>0$, we set $t_{i}:=i T /(n+1)(0 \leq i \leq n+1)$. Let $M_{T}$ be the set of all point configurations with at least one point in each of $C_{i} \times\left(t_{i}, t_{i+1}\right] \times\left[d_{0}, \infty\right) \times\left[0, \beta_{\wedge}\right]$ for all $0 \leq i \leq n$. On $M_{T}, B$ is completely infected at time $T$, and, for $T \rightarrow \infty$, we have $\mathrm{P}\left(M_{T}\right) \rightarrow 1$.

For the proof of part (b), we first show that there is a constant $v>0$ such that

$$
M_{T}:=\left\{B(0,2 t v) \subset Z_{t}^{\cup} \text { for all } t \geq T\right\} \uparrow \mathcal{X} \text { a.s. for } T \rightarrow \infty .
$$

This is basically a consequence of a comparison argument and the shape theorem (Theorem 3 ). Let $f:[0, \infty) \rightarrow[0,1)$ be a bijective increasing function such that $f(x) \leq x$, e.g. $f(x)=$ $x /(1+x)$. The radius distribution $\tilde{\rho}:=\rho \circ f^{-1}$ has bounded support and, thus, satisfies (2). Let $\tilde{Z}$ be the one-type CR model with initial configuration $\Gamma_{\cup}$, growth rate $\beta_{\wedge}$, and radius distribution $\tilde{\rho}$. Theorem 3 gives (5) for $\tilde{Z}$ instead of $Z$. Thus, it suffices to construct a coupling of $Z$ and $\tilde{Z}$ such that $\tilde{Z}_{t} \subset Z_{t}^{\cup}$ for all $t \geq 0$ a.s. Let $Z$ be constructed from $X$, and let $\tilde{Z}$ be 
constructed from $\tilde{X}:=\{(x, s, f(r), w):(x, s, r, w) \in X\}$, scanning from the time of infection, where $X$ is chosen with respect to P. Using this coupling, the desired inclusion easily follows by induction on the times of growth of $\tilde{Z}$. This shows (5).

Suppose that $T$ is chosen so large that $B \subset B(0, T v)$. On $M_{T}$ every point $p=(x, s, r, w)$ producing an effective outburst in $B$ after time $T$ satisfies $r \geq 2 s v-T v \geq s v$ and $w \leq \beta_{\vee}$. More formally, $N_{B, T}^{\text {eff }} \mathbf{1}_{M_{T}} \leq N_{E(B)}$, where $N_{B, T}^{\text {eff }}$ denotes the number of effective outbursts with respect to $Z$ in $B$ after time $T$ and

$$
E(B):=\left\{(x, s, r, w) \in \mathbb{R}_{\times}^{d}: x \in B, r \geq s v, w \leq \beta_{\vee}\right\} .
$$

The P-expectation of $N_{E(B)}$ is

$$
\lambda_{\times}^{d}(E(B))=\int_{B} \mathrm{~d} \lambda^{d}(x) \int \mathrm{d} \rho(r) \int_{0}^{\beta_{\vee}} \mathrm{d} \lambda^{1}(w) \int_{0}^{r / v} \mathrm{~d} \lambda^{1}(s)=\frac{\beta_{\vee} \lambda^{d}(B)}{v} \int \rho(\mathrm{d} r) r,
$$

where the last term is finite by condition (1) on $\rho$. So

$$
\mathrm{P}\left(\left\{N_{B, 0}^{\text {eff }}=\infty\right\} \cap M_{T}\right)=\mathrm{P}\left(\left\{N_{B, T}^{\text {eff }}=\infty\right\} \cap M_{T}\right) \leq \mathrm{P}\left(N_{E(B)}=\infty\right)=0,
$$

i.e. on $M_{T}$ the number of effective outbursts in $B$ is finite a.s., and the result follows from (5).

\subsection{Generalized initial configuration: Lemma 3}

For the proof of (a), we assume that $B$ is some big ball centered at 0 minus the set $B_{\tilde{\Gamma}}$. For rational parameters $c_{0} \in B, t_{0}>0$, and $\delta<d_{0} / 2$, let $L_{c_{0}, \delta}^{t_{0}}$ be the event that $Z_{t_{0}}^{\cup} \cap B$ contains $B\left(c_{0}, \delta\right)$. As $\bigcup_{t_{0}, c_{0}, \delta} L_{c_{0}, \delta}^{t_{0}}=L$, it suffices to show that $\tau_{B}<\infty$ a.s. on $L_{c_{0}, \delta}^{t_{0}}$ for arbitrary $t_{0}$, $c_{0}$, and $\delta$ as above. This follows using the same construction as in the proof of Lemma 2(a), using $C_{0}:=B\left(c_{0}, \delta\right)$.

The proof of part (b) is almost exactly the same as that of part (b) of Lemma 2. Here we let $N_{B, T}^{\text {peff }}$ be the number of virtual effective outbursts in $B$ virtually infecting points outside $B_{\tilde{\Gamma}}$ after time $T$. We can use a comparison argument and Theorem 4 to show that

$$
M_{T}:=\left\{B(0,2 t v) \subset \tilde{Z}_{t} \cup B_{\tilde{\Gamma}} \text { for all } t \geq T\right\} \uparrow L \quad \text { a.s. for } T \rightarrow \infty
$$

for some $v>0$, and $N_{B, T}^{\text {peff }} \mathbf{1}_{M_{T}} \leq N_{E(B)}$ gives $\mathrm{P}\left(\left\{N_{B, 0}^{\text {peff }}=\infty\right\} \cap M_{T}\right)=0$.

\subsection{Generalized shape theorem: Theorem 4}

The basic idea of the proof is to compare the CR model $\tilde{Z}$ with generalized initial configuration $\tilde{\Gamma}$ to the $\mathrm{CR}$ model $Z$ with standard initial configuration $\Gamma:=B_{\tilde{\Gamma}}$, and then to use the shape theorem for $Z$. In order to be able to compare $\tilde{Z}$ and $Z$, we construct them from the same underlying Poisson point process 'scanning from time 0'; see Section 2. For given rational times $0 \leq T_{1} \leq T_{2}$, we define

$$
M_{T_{1}, T_{2}}:=\left\{\zeta_{\Gamma}(Z) \leq T_{1}, Z_{T_{1}} \subset \tilde{Z}_{T_{2}} \cup \Gamma\right\} \cap L,
$$

where $\zeta_{\Gamma}(Z)$ is the time of the last effective outburst in $\Gamma$ with respect to $Z$. Here $Z_{T_{1}}$ is bounded, and, by Lemmas 2 and 3, both $\zeta_{\Gamma}(Z)$ and $\tau_{B-\Gamma}(\tilde{Z})$ are finite on $L$ for any bounded Borel set $B$, where $\tau_{B-\Gamma}(\tilde{Z})$ is the first time that $B$ is infected with respect to $\tilde{Z}$. Thus, $\bigcup_{T_{1}, T_{2}} M_{T_{1}, T_{2}}=L$, so it suffices to show the generalized shape theorem on $M_{T_{1}, T_{2}}$. By the shape theorem for $Z$, there exists a $\mu>0$ independent of $\Gamma, \tilde{\Gamma}$, and $\beta$ such that

$$
\left(1-\frac{\varepsilon}{2}\right) B\left(0, \beta \mu^{-1}\right) \subset \frac{Z_{t}}{t} \subset\left(1+\frac{\varepsilon}{2}\right) B\left(0, \beta \mu^{-1}\right) \quad \text { for sufficiently large } t .
$$


In order to see that this implies that

$$
(1-\varepsilon) B\left(0, \beta \mu^{-1}\right) \subset \frac{\tilde{Z}_{t} \cup \Gamma}{t} \subset(1+\varepsilon) B\left(0, \beta \mu^{-1}\right) \quad \text { for sufficiently large } t
$$

on $M_{T_{1}, T_{2}}$, we use the facts that $\left(t-T_{2}\right) / t \geq(1-\varepsilon) /(1-\varepsilon / 2)$ for sufficiently large $t$ and

$$
\tilde{Z}_{t} \cup \Gamma \subset Z_{t} \subset \tilde{Z}_{t+T_{2}} \cup \Gamma \text { for all } t \geq 0 \text { on } M_{T_{1}, T_{2}} .
$$

For the proof of (6), we fix a point configuration $X \in M_{T_{1}, T_{2}}$. We note that the chosen coupling of $\tilde{Z}$ and $Z$ implies that every $p=(x, s, r, w) \in X$ with $x \notin \Gamma$ and $w \leq \beta$ produces an outburst in both models, and in both models the time between the infection of $x$ and the time $p$ produces the outburst is $s$.

For the inclusion $\tilde{Z}_{t} \cup \Gamma \subset Z_{t}$, we observe that $Z_{t} \supset \Gamma$ is trivial and $\tilde{Z}_{t} \subset Z_{t}$ can be shown by induction on the growth times of $\tilde{Z}$. For $t=0$, the assertion is trivial. If $t>0$ is a growth time then either this growth is from the initial configuration $\tilde{\Gamma}$ of $\tilde{Z}$ (and in this case $\tilde{Z}_{t} \subset Z_{t}$ follows from $\left.\Gamma \subset Z_{t}\right)$ or there is a point $p=(x, s, r, w) \in X$ producing an outburst at time $t$ with respect to $\tilde{Z}$. In this case $w \leq \beta$ and $x$ was infected in $\tilde{Z}$ at time $t^{\prime}=t-s$. By the induction hypothesis, $\tilde{Z}_{t^{\prime}} \subset Z_{t^{\prime}}$, so $x$ was infected in $Z$ at an earlier time $t^{\prime \prime} \leq t^{\prime}$, i.e. $p$ produces an outburst with respect to $Z$ at time $t^{\prime \prime}+s \leq t^{\prime}+s=t$.

For $Z_{t} \subset \tilde{Z}_{t+T_{2}} \cup \Gamma$, we argue with induction on the growth times of $Z$. For $0 \leq t \leq T_{1}$, the assertion is trivial since $Z_{T_{1}} \subset \tilde{Z}_{T_{2}} \cup \Gamma$ on $M_{T_{1}, T_{2}}$. If $t>T_{1}$ is a growth time with respect to $Z$, there is a point $p=(x, s, r, w)$ producing an effective outburst in $Z$ at time $t$. So $x$ was infected in $Z$ at time $t^{\prime}=t-s<t$, which gives $x \in Z_{t^{\prime}} \subset \tilde{Z}_{t^{\prime}+T_{2}} \cup \Gamma$ by the induction hypothesis. On $M_{T_{1}, T_{2}}$ the last effective outburst located in $\Gamma$ occurs before $T_{1}$. Since $t>T_{1}$, we know that $x \notin \Gamma$, so $x \in \tilde{Z}_{t^{\prime}+T_{2}} \cup \Gamma$ implies that $x \in \tilde{Z}_{t^{\prime}+T_{2}}$. Thus, the outburst caused by $p$ with respect to $\tilde{Z}$ occurs no later than $\left(t^{\prime}+T_{2}\right)+s=t+T_{2}$.

\section{Proof of the main result: Theorem 1}

\subsection{Outline of the strategy}

We denote the two given CR models by $Z(\Gamma)$ and $Z\left(\Gamma^{\prime}\right)$. Assuming that

$$
\mathrm{P}(G(\Gamma))>0 \quad \text { and } \quad \mathrm{P}\left(L_{i}\left(\Gamma^{\prime}\right)\right)>0 \quad \text { for } i=1,2,
$$

it suffices to show that $\mathrm{P}\left(G\left(\Gamma^{\prime}\right)\right)>0$. Without loss of generality, we assume that $\beta_{1} \geq \beta_{2}$. Our basic strategy to show that $\mathrm{P}\left(G\left(\Gamma^{\prime}\right)\right)>0$ in the next subsections will be to decompose the evolution of $Z(\Gamma)$ into an initial part and a final part separated by a space-dependent time horizon. This will be chosen such that, with positive probability, the initial evolution will infect certain fundamental regions before the time horizon, and in the final evolution outbursts in these fundamental regions will yield infinite infection paths for both types after the time horizon. After that we will describe an initial evolution of $Z\left(\Gamma^{\prime}\right)$ infecting the fundamental regions before the time horizon and having positive probability. By coupling the final evolution of $Z\left(\Gamma^{\prime}\right)$ to the final evolution of $Z(\Gamma)$ we also have coexistence for the initial condition $\Gamma^{\prime}$, i.e. $\mathrm{P}\left(\Gamma^{\prime}\right)>0$.

\subsection{Escaping the initial configuration in $Z\left(\Gamma^{\prime}\right)$}

By (7), the initial configuration $\Gamma^{\prime}$ allows each type to escape some ball with positive probability. However, what we need is that $\Gamma^{\prime}$ also allows both types to escape some large ball at the same time with positive probability. To describe these escape routes, we need some notation for line segments. For given points $x_{1} \neq x_{2}$, let $l_{x_{1} x_{2}}$ denote the straight line passing 
through these points. We identify $l_{x_{1} x_{2}}$ with $\mathbb{R}$ such that $x_{1}<x_{2}$ so that we can use the induced order on $l_{x_{1} x_{2}}$ along with the corresponding interval notation for line segments.

Definition 2. We say that an infection configuration $\left(A_{1}, A_{2}\right)$ has escape corridors specified by $x_{1}, x_{2} \in \mathbb{R}^{d}$ and $r_{0}, \delta_{0}>0$ whenever $d\left(x_{1}, x_{2}\right)>d_{0}+4 \delta_{0}, B\left(x_{i}, \delta_{0}\right) \subset A_{i} \subset B\left(0, r_{0}\right)$, $\left(-\infty, x_{1}\right]_{+\delta_{0}} \cap A_{2}=\varnothing$, and $\left[x_{2}, \infty\right)_{+\delta_{0}} \cap A_{1}=\varnothing$.

We note that in the situation of Definition 2 we can use Lemma 1 to let the two species grow along $\left(-\infty, x_{1}\right]$ and $\left[x_{2}, \infty\right)$, and we will show that this situation occurs with positive probability.

Lemma 4. Let $d_{0}>0$ be the constant chosen in (4). For suitably chosen $T_{0}^{\prime} \geq 0, x_{1}, x_{2} \in \mathbb{R}^{d}$, $\delta_{0}<d_{0} / 8$, and $r_{0}>2 d_{0}$, we have $\mathrm{P}\left(M_{0}^{\prime}\right)>0$, where $M_{0}^{\prime}$ is the event that $Z_{T_{0}^{\prime}}\left(\Gamma^{\prime}\right)$ has escape corridors specified by $x_{1}, x_{2}, r_{0}$, and $\delta_{0}$.

\subsection{Time horizon and fundamental regions for $Z(\Gamma)$}

In the following we will define subsets of $M_{0}:=G(\Gamma)$ by specifying certain parameters of the infection evolution of $Z(\Gamma)$. After that we will show that the parameters can be chosen in a way such that these subsets of $M_{0}$ have positive probability. In step 1 we use the parameters $r_{0}$ and $T_{0}^{\prime}$ chosen in Lemma 4 . In step 3 we restrict our attention to only one infinite infection path of the stronger type. This idea was already used by Deijfen et al. [3] for the two-type discrete Richardson model.

Step 1. Let $r_{2}>r_{1}>r_{0}$ and $B_{i}:=B\left(0, r_{i}\right)$. Let $M_{1}$ be the set of configurations of $M_{0}$ such that

- at time $T_{0}^{\prime}$ the infected region is contained in $B_{1}$,

- no outburst with position in $B_{1}$ infects anything outside $B_{2}$.

Step 2. Let $d_{1}>0$. Let $M_{2}$ be the set of configurations of $M_{1}$ such that $2 d_{1}$ is a lower bound on the distances of the positions of effective outbursts in $B_{2}$ to

- the lines passing through the position of another effective outburst in $B_{2}$ and the origin,

- the boundaries of infection balls of outbursts that infect regions in $B_{2}$,

- the boundaries of the balls $B_{1}$ and $B_{2}$.

Step 3. Let $0<d_{2}<d_{1}$, let $D^{1}, D_{1}^{2}, \ldots, D_{k}^{2}$ be balls of radius $d_{2}$ ('fundamental balls'), and let $T\left(D^{1}\right), T\left(D_{1}^{2}\right), \ldots, T\left(D_{k}^{2}\right)>0$ be times associated to the balls. Let $M_{3}$ be the set of configurations of $M_{2}$ such that

- $D^{1}$ contains the starting point of an infinite type- 1 infection path that never returns to $B_{2}$,

- the effective outbursts of type 2 within $B_{2}-B_{1}$ are located in the balls $D_{1}^{2}, \ldots, D_{k}^{2}$, $k \geq 1$ (one ball for each outburst),

- at the time $T(\cdot)$ corresponding to a fundamental ball, the position of the corresponding outburst is infected, but the outburst has not yet occurred.

Lemma 5. The above parameters $r_{1}, r_{2}, d_{1}, d_{2}, D^{1}, \ldots, D_{k}^{2}$, and $T\left(D^{1}\right), \ldots, T\left(D_{k}^{2}\right)$ can be chosen so that $\mathrm{P}\left(M_{i}\right)>0$ for $i=1,2,3$; furthermore,

$$
r_{1}>r_{0}+4 d_{0}, \quad r_{2}>r_{1}+4 d_{0}, \quad d_{1}<\frac{d_{0}}{4}, \quad d_{2}<\min \left\{\frac{d_{1}}{10}, \frac{d_{1}^{2}}{8 d_{0}}\right\} .
$$


We now choose the parameters according to the above lemma, and consider them to be fixed for the rest of the proof. Let $M_{\mathrm{in}, \mathrm{fi}}:=M_{3}$ be the desired specification of the (initial and final) infection evolution of $Z(\Gamma)$. We have $\mathrm{P}\left(M_{\mathrm{in}, \mathrm{fi}}\right)>0$ by Lemma 5 , and on $M_{\mathrm{in}, \mathrm{fi}}$ the following properties hold.

- All fundamental balls are contained in $B_{2}-B_{1}$ and keep a distance of at least $2 d_{1}-d_{2}$ to the boundaries of this set. All type-2 fundamental balls keep a distance of at least $2 d_{1}-d_{2}$ to the line through the center of $D^{1}$ and the origin. The distance between two fundamental balls is at least $2 d_{1}-2 d_{2}$. The times corresponding to the fundamental balls are greater than $T_{0}^{\prime}$.

- Each fundamental ball is infected as a whole (so the type-1 fundamental ball is completely 1 -infected and the type- 2 fundamental balls are completely 2 -infected), and each fundamental ball contains the position of exactly one effective outburst. A fundamental ball is infected at the associated time, but the effective outburst in this ball occurs later.

- Every 2-infection of a region in $B_{2}^{\mathrm{c}}$ originating in $B_{2}$ in fact originates in one of the type-2 fundamental balls. The type- 1 fundamental ball is the starting point of an infection path to infinity for type 1 such that this path never returns to $B_{2}$.

In Section 4.1 we introduced the notion of a space-dependent time horizon. We now choose the time horizon on the fundamental balls to be the times associated with these balls, and on $B_{2}^{\mathrm{c}}$ to be $T\left(B_{2}^{\mathrm{c}}\right):=0$. On the rest of the space, it will be chosen later.

\subsection{Initial evolution of $Z\left(\Gamma^{\prime}\right)$}

In this section we only consider the model with initial configuration $\Gamma^{\prime}$, so we set $Z:=Z\left(\Gamma^{\prime}\right)$. We would like to construct an initial evolution starting from $\Gamma^{\prime}$ that infects all fundamental balls before the time horizon. More precisely, we define $T^{\prime}:=\min \left\{T\left(D^{1}\right), T\left(D_{1}^{2}\right), \ldots, T\left(D_{k}^{2}\right)\right\}>$ $T_{0}^{\prime}$ and $M_{\text {in }}^{\prime}$ to be the set of all configurations such that, for $Z$ at time $T^{\prime}$, ball $D^{1}$ is 1-infected, all balls $D_{i}^{2}(1 \leq i \leq k)$ are 2-infected, and no point of $B_{2}^{\mathrm{c}}$ is infected. By definition, $M_{\text {in }}^{\prime}$ depends only on the point configuration in $\Delta_{\text {in }}:=B_{2} \times\left[0, T^{\prime}\right]$. In order to show that $\mathrm{P}\left(M_{\text {in }}^{\prime}\right)>0$, we give a step-by-step description of a suitable infection evolution with the desired properties. The main reason why this explicit construction is possible is that we only have to infect one small ball with type 1 , but are allowed to infect everything else in $B_{2}$ with type 2 . For the construction, we choose fixed times $T_{0}^{\prime}<T_{1}^{\prime}<\cdots<T_{4}^{\prime}:=T^{\prime}$, and we define a sequence of events $M_{i}^{\prime}$ describing a desirable infection state at time $T_{i}^{\prime}$.

We will use interval notation for line segments of $l_{0 a}$, where $a$ denotes the center of $D^{1}$. Furthermore, let $c_{1}$ denote the point on $[0, \infty) \subset l_{0 a}$ with $d\left(c_{1}, 0\right)=\left(r_{0}+r_{1}\right) / 2, c_{2}:=-c_{1}$, $C:=B_{2}-B_{1}-\left(\partial B_{2}\right)_{+d_{0}+d_{2}}-[0, \infty)_{+d_{0}+2 d_{2}}$, and $C_{+}:=B_{2}-B_{1}-\left(\partial B_{2}\right)_{+d_{1}}-[0, \infty)_{+d_{1}}$; see Figure 1. We note that $D^{1} \subset[0, \infty)_{+d_{1}}$ and, thus, $C_{+} \cap D^{1}=\varnothing$. The events $M_{i}^{\prime}$ (as shown in Figure 2) can now be defined by

$$
\begin{aligned}
M_{1}^{\prime} & :=\left\{B\left(c_{1}, d_{2}\right) \subset Z_{T_{1}^{\prime}}^{1} \subset B_{1}-\left[-r_{1}, c_{2}\right]_{+d_{2}}, B\left(c_{2}, d_{2}\right) \subset Z_{T_{1}^{\prime}}^{2} \subset B_{1}-\left[c_{1}, r_{1}\right]_{+d_{2}}\right\}, \\
M_{2}^{\prime} & :=\left\{B\left(c_{1}, d_{2}\right) \subset Z_{T_{2}^{\prime}}^{1} \subset B_{1}, C_{+d_{0}-d_{2}}-B_{1} \subset Z_{T_{2}^{\prime}}^{2} \subset B_{2}-\left[c_{1}, r_{2}\right]_{+d_{2}}\right\}, \\
M_{3}^{\prime} & :=\left\{D^{1} \subset Z_{T_{3}^{\prime}}^{1} \subset B_{1} \cup\left[0, r_{2}\right]_{+d_{1}} \cap B_{2}, C_{+d_{0}-d_{2}}-B_{1} \subset Z_{T_{3}^{\prime}}^{2} \subset B_{2}\right\}, \\
M_{4}^{\prime} & :=\left\{D^{1} \subset Z_{T_{4}^{\prime}}^{1} \subset B_{2}, C_{+} \subset Z_{T_{4}^{\prime}}^{2} \subset B_{2}\right\} ;
\end{aligned}
$$

$M_{0}^{\prime}$ is the event of positive probability chosen in Lemma 4. 

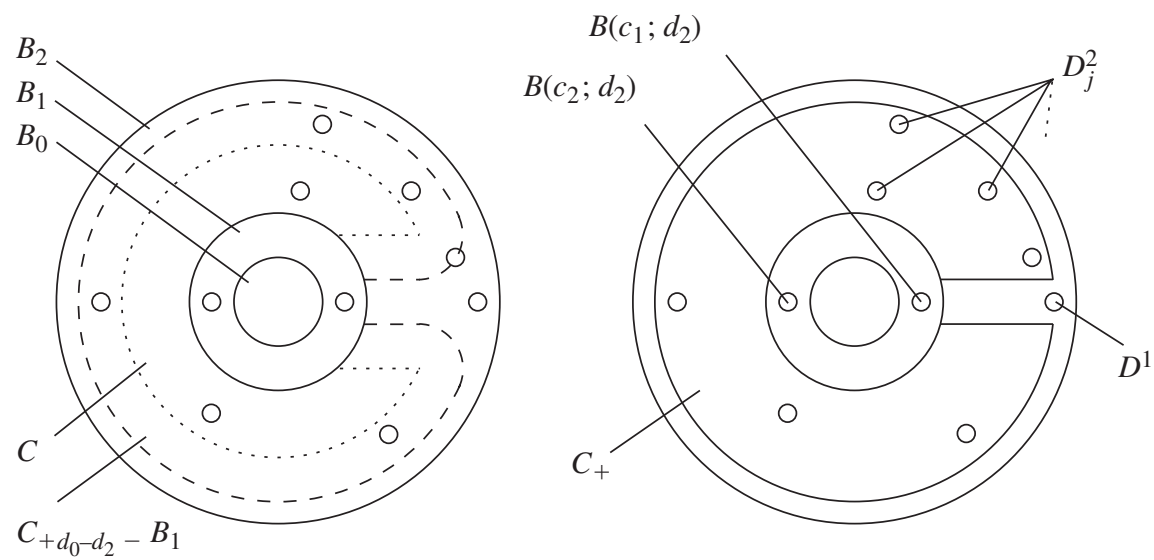

FIGURE 1: Sketch of the sets relevant in the definition of the initial evolution.
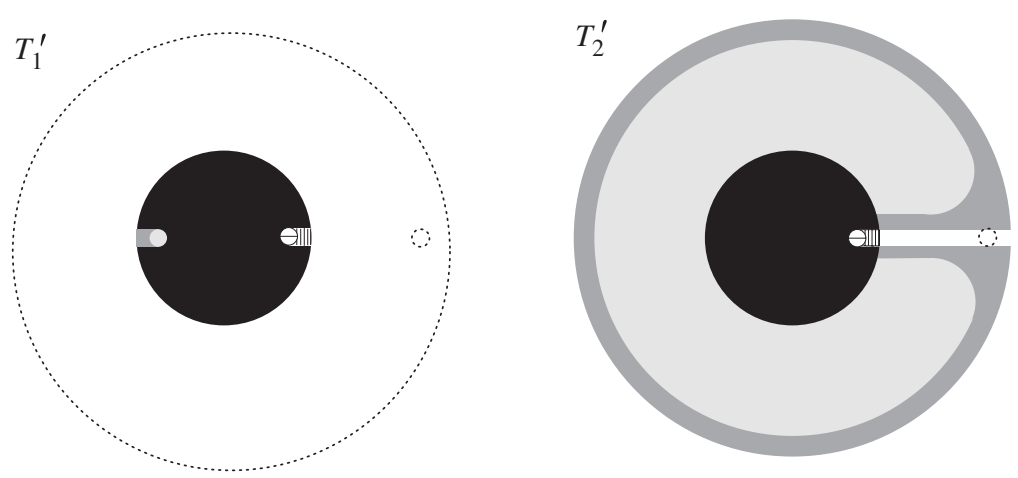

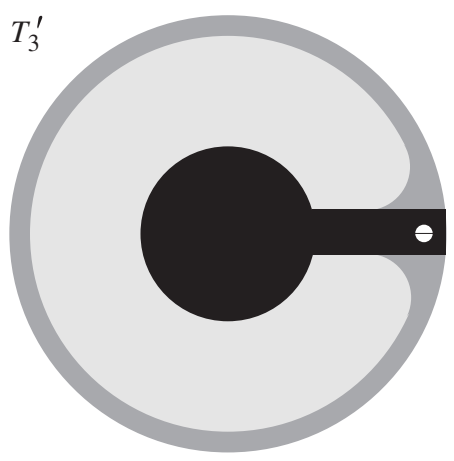

Infected by species 1 Infected by species 2 Not infected

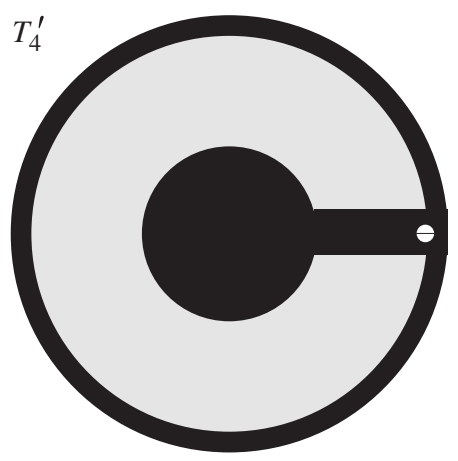

盂 Infected by species 1 or not at all Infected by species 2 or not at all - Infection state unspecified

FIgURE 2: Sketch of the infection states at time $T_{i}^{\prime}$ on the set $M_{i}^{\prime}$ for $1 \leq i \leq 4$.

Lemma 6. There are events $M_{(i-1) \rightarrow i}^{\prime}(i=1,2,3,4)$ describing an infection evolution in $\left(T_{i-1}^{\prime}, T_{i}^{\prime}\right]$ such that $\mathrm{P}\left(M_{(i-1) \rightarrow i}^{\prime}\right)>0$ and $M_{i-1}^{\prime} \cap M_{(i-1) \rightarrow i}^{\prime} \subset M_{i}^{\prime}$. 
By the independence property of the Poisson process, $\mathrm{P}\left(M_{i-1}^{\prime} \cap M_{(i-1) \rightarrow i}^{\prime}\right)=\mathrm{P}\left(M_{i-1}^{\prime}\right) \times$ $\mathrm{P}\left(M_{(i-1) \rightarrow i}^{\prime}\right)$, so Lemma 6 inductively gives $\mathrm{P}\left(M_{i}^{\prime}\right)>0$ for $i=0, \ldots, 4$. As, by construction, all balls $D_{i}^{2}$ are contained in $C_{+}$, we have $M_{4}^{\prime} \subset M_{\text {in }}^{\prime}$ and, thus,

$$
\mathrm{P}\left(M_{\mathrm{in}}^{\prime}\right)>0 \text {. }
$$

\subsection{Final evolution of $Z\left(\Gamma^{\prime}\right)$}

Let us now consider the two-type $\mathrm{CR}$ model $\tilde{Z}=Z(\tilde{\Gamma})$ with generalized initial configuration $\tilde{\Gamma}$ consisting of all fundamental balls with their associated times and types, and $D_{0}$, the remaining part of $B_{2}$, with associated time $\infty$. We assume that $\tilde{Z}$ is constructed using the Poisson point process underlying $Z(\Gamma)$. On $M_{\mathrm{in}, \mathrm{fi}}$ the infection evolution of $\tilde{Z}$ in $B_{2}^{\mathrm{c}}$ is very similar to that of $Z(\Gamma)$, differing only in that type 1 has been reduced considerably. However, we will show that in $\tilde{Z}$ type 1 is still strong enough to grow without bound. Let $M_{\mathrm{fi}}:=G(\tilde{\Gamma})$ denote the event that in $\tilde{Z}$ both types grow without bounds.

Lemma 7. We have $M_{\mathrm{in}, \mathrm{fi}} \subset M_{\mathrm{fi}}$ and, thus, $\mathrm{P}\left(M_{\mathrm{fi}}\right)>0$.

We would now like to use $M_{\mathrm{fi}}$ for the final evolution of $Z\left(\Gamma^{\prime}\right)$, but unfortunately on $M_{\mathrm{in}}^{\prime} \cap M_{\mathrm{fi}}$ we are not guaranteed to have coexistence for $Z\left(\Gamma^{\prime}\right)$. The problem is that in the construction of $\tilde{Z}$ from a point configuration all points in $D^{0}$ are ignored, so $M_{\mathrm{fi}}$ has no information on points in this region, whereas, using $M_{\text {in }}^{\prime}$ for the initial evolution of $Z\left(\Gamma^{\prime}\right)$, it is very likely that $D^{0}$ will be at least partially infected at time $T^{\prime}$. To guarantee coexistence in $Z\left(\Gamma^{\prime}\right)$, we would thus like to delete any point of the underlying point configuration in $D^{0}$, producing a virtual effective outburst virtually infecting points of $B_{2}^{\mathrm{c}}$ after time $T^{\prime}$. By Lemma 3 we know that $\tilde{\zeta}_{B_{2}}<\infty$ a.s. on $M_{\mathrm{fi}}$, because $M_{\mathrm{fi}} \subset L$. Thus, we can choose a time $T^{\prime \prime}>T^{\prime}$ such that,

$$
\text { for } M_{\mathrm{fi}}^{\prime}:=M_{\mathrm{fi}} \cap\left\{\tilde{\zeta}_{B_{2}}<T^{\prime \prime}\right\} \text {, we still have } \mathrm{P}\left(M_{\mathrm{fi}}^{\prime}\right)>0 \text {, }
$$

where $M_{\mathrm{fi}}^{\prime}$ denotes the final evolution in the $\Gamma^{\prime}$-model. Setting $T\left(D^{0}\right):=T^{\prime \prime}$ we have defined a time horizon on all of $\mathbb{R}^{d}$. Since $M_{\mathrm{fi}}^{\prime}$ is defined in terms of $\tilde{Z}$, it depends only on part of the point configuration after the time horizon, i.e. on the point configuration in

$$
\Delta_{\mathrm{fi}}:=\bigcup_{D \in \mathscr{D}} D \times[T(D), \infty), \quad \text { where } \mathscr{D}=\left\{B_{2}^{\mathrm{c}}, D^{0}, D^{1}, D_{1}^{2}, \ldots, D_{k}^{2}\right\} .
$$

We now delete the remaining points that could interfere with the desirable final evolution. Let $M_{\mathrm{de}}^{\prime}$ be the set of all point configurations without any point in

$$
\Delta_{\mathrm{de}}:=\bigcup_{D \in \mathcal{D}} D \times\left[T^{\prime}, T(D)\right) \times \mathbb{R}_{+} \times\left[0, \beta_{1}\right] .
$$

As $T\left(B_{2}^{\mathrm{c}}\right)=0$, we have $\lambda_{\times}^{d}\left(\Delta_{\mathrm{de}}\right)<\infty$, and, thus,

$$
\mathrm{P}\left(M_{\mathrm{de}}^{\prime}\right)>0 \text {. }
$$

By construction, the sets of point configurations $M_{\mathrm{in}}^{\prime}, M_{\mathrm{de}}^{\prime}$, and $M_{\mathrm{fi}}^{\prime}$ depend on $\Delta_{\mathrm{in}}, \Delta_{\mathrm{de}}$, and $\Delta_{\mathrm{fi}}$, respectively, and these sets are disjoint. By the independence property of the Poisson process, we thus have

$$
\mathrm{P}\left(M_{\mathrm{in}, \mathrm{fi}}^{\prime}\right)=\mathrm{P}\left(M_{\mathrm{in}}^{\prime}\right) \mathrm{P}\left(M_{\mathrm{de}}^{\prime}\right) \mathrm{P}\left(M_{\mathrm{fi}}^{\prime}\right)>0 \quad \text { for } M_{\mathrm{in}, \mathrm{fi}}^{\prime}:=M_{\mathrm{in}}^{\prime} \cap M_{\mathrm{de}}^{\prime} \cap M_{\mathrm{fi}}^{\prime},
$$

where all probabilities are positive because of (9), (10), and (11). It is an immediate consequence of the construction that on $M_{\text {in,fi }}^{\prime}$ the model $Z\left(\Gamma^{\prime}\right)$ has exactly the same infection evolution in $B_{2}^{\mathrm{c}}$ as the model $\tilde{Z}$, and we have coexistence for the model $\tilde{Z}$ on $M_{\mathrm{in,fi}}^{\prime}$ since $M_{\mathrm{in}, \mathrm{fi}}^{\prime} \subset M_{\mathrm{fi}}^{\prime} \subset M_{\mathrm{fi}}$. This implies that we also have coexistence for the model $Z$ on $M_{\text {in,fi }}^{\prime}$, i.e. $\mathrm{P}\left(G\left(\Gamma^{\prime}\right)\right)>0$. 


\section{Proofs of the lemmas}

\subsection{Escape corridors: Lemma 4}

In this subsection we only consider the $\mathrm{CR}$ model with initial configuration $\Gamma^{\prime}$, so let $Z:=$ $Z\left(\Gamma^{\prime}\right) ; Z$ does not change a.s. if $\Gamma^{\prime}$ is modified outside its support. Thus, we may assume without loss of generality that $\operatorname{supp}\left(\Gamma_{i}^{\prime}\right)=\overline{\Gamma_{i}^{\prime}}$, which implies that $\operatorname{supp}\left(Z_{i}^{t}\right)=\overline{Z_{i}^{t}}$ for all $t \geq 0$. The proof of Lemma 4 consists of three parts. First we construct an event $M_{0}^{\prime \prime}$ of positive probability such that on $M_{0}^{\prime \prime}$ there is a time $T$ and distinct $y_{i} \in \overline{Z_{T}^{i}}(i=1,2)$ such that, using interval notation on $l_{y_{1} y_{2}}$,

$$
\left(-\infty, y_{1}-d_{0}\right] \cap \overline{Z_{T}^{2}}=\varnothing \quad \text { and } \quad\left[y_{2}+d_{0}, \infty\right) \cap \overline{Z_{T}^{1}}=\varnothing .
$$

In the second part we show that a stronger version of (12) holds for fixed values of $T, y_{1}$, and $y_{2}$ with positive probability. In the third part we verify the existence of deterministic escape corridors.

For the first part, we define the minimal enclosing ball (MEB) of a set $K \subset \mathbb{R}^{d}$ to be a closed ball containing $K$ with minimal radius. Every bounded set has a unique MEB. We will make repeated use of the following property of MEBs.

(A) The MEB $B$ of a compact set $K$ is also the MEB of $\partial B \cap K$.

Let $B=B(m, r)$ denote the MEB of $\overline{\Gamma_{1}^{\prime}} \cup \overline{\Gamma_{2}^{\prime}}$. Without loss of generality, $\partial B \cap \overline{\Gamma_{1}^{\prime}} \neq \varnothing$ and $\partial B \cap \overline{\Gamma_{2}^{\prime}}=\varnothing$. (By (A), one of these sets is nonempty. If the other set is nonempty, we can argue similarly. If both are nonempty, the assertion is trivial as we can choose $T=0$ and $y_{i} \in \partial B \cap \overline{\Gamma_{i}^{\prime}}$.) Thus, by (A), $B$ is the MEB of $\partial B \cap \overline{\Gamma_{1}^{\prime}}$.

Case 1: $\rho([r, \infty))>0$. In this case we assume without loss of generality that $d_{0}$ with property (4) was chosen such that $d_{0} \geq r$. We set $M_{0}^{\prime \prime}:=\mathcal{X}, T=0$, and choose $y_{2} \in$ $\overline{\Gamma_{2}^{\prime}}-\{m\}$ and $y_{1} \in \partial B \cap \overline{\Gamma_{1}^{\prime}}$ such that $d\left(y_{1}, y_{2}\right)>r$. (As $B(m, r)$ is the MEB of $\partial B \cap \overline{\Gamma_{1}^{\prime}}$, $B\left(y_{2}, r\right)$ does not enclose this set.) In order to see that $y_{1}$ and $y_{2}$ have the above property, we only have to check that $\left[y_{2}+d_{0}, \infty\right) \cap \overline{\Gamma_{1}^{\prime}} \subset\left[y_{2}+d_{0}, \infty\right) \cap B=\varnothing$, which follows from $\lambda^{1}\left(l_{y_{1} y_{2}} \cap B-\left[y_{1}, y_{2}\right]\right)<2 r-r=r \leq d_{0}$.

Case 2: $\rho([r, \infty))=0$. Let $M_{1}^{\prime \prime}:=\left\{Z_{T^{\prime \prime}}^{1}=\Gamma_{1}^{\prime}\right\}$ and $M_{2}^{\prime \prime}=\left\{Z_{T^{\prime \prime}}^{2} \not \subset B\right\}$, where $T^{\prime \prime}$ is chosen sufficiently large such that $\mathrm{P}\left(M_{2}^{\prime \prime}\right)>0$. We also have $\mathrm{P}\left(M_{1}^{\prime \prime}\right)>0$, and, since the absence of outbursts of type 1 can only strengthen type 2 , we have $\mathrm{P}\left(M_{2}^{\prime \prime} \mid M_{1}^{\prime \prime}\right)>\mathrm{P}\left(M_{2}^{\prime \prime}\right)$. Setting $M_{0}^{\prime \prime}=M_{1}^{\prime \prime} \cap M_{2}^{\prime \prime}$, we obtain $\mathrm{P}\left(M_{0}^{\prime \prime}\right)>0$. On $M_{0}^{\prime \prime}$ at some time $T$ an outburst $B^{\prime}=B\left(x^{\prime}, r^{\prime}\right)$ of species 2 occurs which for the first time infects a region in $B^{\mathrm{c}}$. As $r^{\prime}<r$ a.s. and as $B$ is the MEB of $\partial B \cap \overline{\Gamma_{1}^{\prime}}, B^{\prime}$ cannot enclose this set. So we can choose points $y_{1} \in \partial B \cap \overline{\Gamma_{1}^{\prime}}-B^{\prime}$ and $y_{2} \in \partial B \cap B^{\prime}$ that have the above property.

For the second part, we note that the infection configuration is always constant over a time interval of positive length, $\overline{Z_{T}^{i}}$ is always a compact set, the infected region is always bounded, and if the points $y_{i}^{\prime}$ are sufficiently close to $y_{i}$, these points still satisfy (12). This shows that on $M_{0}^{\prime \prime}$ there are rational parameters $T^{\prime}>0,0<\delta<d_{0} / 8, y_{1}^{\prime}, y_{2}^{\prime} \in \mathbb{R}^{d}$, and $r>0$ such that

$$
\begin{gathered}
\left(-\infty, y_{1}^{\prime}-d_{0}\right]_{+4 \delta} \cap \overline{Z_{T^{\prime}}^{2}}=\varnothing, \quad\left[y_{2}^{\prime}+d_{0}, \infty\right)_{+4 \delta} \cap \overline{Z_{T^{\prime}}^{1}}=\varnothing, \quad d\left(y_{1}^{\prime}, y_{2}^{\prime}\right)>4 \delta, \\
Z_{T^{\prime}}^{\cup} \subset B(0, r), \quad \text { and } \quad \lambda^{d}\left(B\left(y_{i}^{\prime}, \delta\right) \cap Z_{T^{\prime}}^{i}\right)>0 \quad \text { for } i=1,2 .
\end{gathered}
$$

Thus, for some particular choice of these parameters, the event $M_{00}^{\prime}$ for which (13) holds for this choice of parameters has positive probability. 
Finally, for the last part, let $M_{01}^{\prime}$ be the set of all point configurations with exactly one point in each of the sets

$$
\left(B\left(y_{i}^{\prime}, \delta\right) \cap Z_{T^{\prime}}^{i}\right) \times\left(T^{\prime}, T^{\prime}+1\right] \times\left[d_{0}, d_{0}+\delta\right) \times\left[0, \beta_{2}\right], \quad i=1,2,
$$

and no additional points in $B(0, r) \times\left(T^{\prime}, T^{\prime}+1\right] \times\left[0, \beta_{1}\right]$. Note that $M_{01}^{\prime} \in \mathcal{F}_{\mathbb{R}^{d} \times\left(T^{\prime}, T^{\prime}+1\right]}$ is independent of $M_{00}^{\prime} \in \mathcal{F}_{\mathbb{R}^{d} \times\left[0, T^{\prime}\right]}$ and has positive probability. As a consequence $M_{0}^{\prime}:=$ $M_{00}^{\prime} \cap M_{01}^{\prime}$ has positive probability. On $M_{0}^{\prime}$ the infection configuration $Z_{T^{\prime}+1}$ has escape corridors specified by the parameters $x_{1}:=y_{1}^{\prime}-d_{0}+2 \delta, x_{2}:=y_{2}^{\prime}+d_{0}-2 \delta, r_{0}:=r+2 d_{0}$, and $\delta_{0}:=\delta$.

\subsection{Specifying the evolution in the $\boldsymbol{\Gamma}$-model: Lemma 5}

Step 1. We have $M_{0} \cap\left\{Z_{T_{0}^{\prime}} \subset B\left(0, r_{1}\right)\right\} \cap\left\{\zeta_{B_{1}}<T\right\} \cap\left\{Z_{T} \subset B\left(0, r_{2}\right)\right\} \subset M_{1}$ for any $T>0$. The events $\left\{Z_{T_{0}^{\prime}} \subset B\left(0, r_{1}\right)\right\},\left\{\zeta_{B_{1}}<T\right\}$, and $\left\{Z_{T} \subset B\left(0, r_{2}\right)\right\}$ are increasing in $r_{1}, T$, and $r_{2}$, respectively, and $\zeta_{B_{1}}<\infty$ a.s. by Lemma 2 and, for fixed $T, Z_{T}$ is bounded a.s. Thus, we only have to choose first $r_{1}$, then $T$, and then $r_{2}$ large enough in order to obtain $\mathrm{P}\left(M_{1}\right)>0$.

Step 2. Observing that the event of all given distances being bounded from below by $2 d_{1}$ is decreasing in $d_{1}$, it remains to show that the infimum of the distances is positive a.s. Since every single distance is positive a.s., it suffices to observe that the number of effective outbursts in $B_{2}$ is finite a.s. by Lemma 2 and the number of outbursts that infect a region in $B_{2}$ is finite a.s., as $B_{2}$ is completely infected after a finite time by Lemma 2 . Therefore, if we choose $d_{1}>0$ small enough, we have $\mathrm{P}\left(M_{2}\right)>0$.

Step 3. We choose a small $d_{2}>0$ satisfying (8), and we fix a finite covering of $B_{2}$ consisting of balls of radius $d_{2}$. For any infection configuration, it is possible to choose a finite number of balls with the properties described in step 3 of Section 4.3, and as in each ball the time span between the infection of the outburst position and the outburst at that position is positive a.s., we can choose a rational time to serve as the time horizon on the ball. The number of ways to choose a finite set of fundamental balls from the finite covering and a rational time horizon is countable, so there is a specific choice of fundamental balls and corresponding time horizons such that $\mathrm{P}\left(M_{3}\right)>0$.

\subsection{Local evolution in the $\Gamma^{\prime}$-model: Lemma 6}

$M_{0 \rightarrow 1}^{\prime}$ : infecting the balls $B\left(c_{i}, d_{2}\right)$. We use the parameters chosen in Lemma $4, \delta:=\delta_{0}$, and $T_{1 / 2}^{\prime}:=\left(T_{0}^{\prime}+T_{1}^{\prime}\right) / 2$. Choosing two curves $C_{i} \subset B_{1 / 2}:=B\left(0, r_{0}+2 d_{0}\right)(i=1,2)$ joining $x_{i} \in B_{0}$ and $c_{i} \in \partial B_{1 / 2}$, we use Lemma 1 to let type 1 grow along $C_{1}$ in the time interval $\left(T_{0}^{\prime}, T_{1 / 2}^{\prime}\right]$ with precision $\delta$ and after that to let type 2 grow along $C_{2}$ in the time interval $\left(T_{1 / 2}^{\prime}, T_{1}^{\prime}\right]$ with precision $\delta$. This infection evolution defines an event $M_{0 \rightarrow 1}^{\prime}$ with the desired properties, provided that the curves $C_{i}$ satisfy

$$
d\left(C_{1}, Z_{T_{0}^{\prime}}^{2}\right)>\delta, \quad d\left(C_{2}, Z_{T_{0}^{\prime}}^{1}\right)>\delta, \quad d\left(C_{1}, C_{2}\right)=d\left(x_{1}, x_{2}\right)>d_{0}+4 \delta,
$$

and

$$
d\left(C_{1}, B\left(c_{2}, d_{2}\right)\right)>d_{0}+2 \delta .
$$

For a possible construction of such curves, see Figure 3. Here $b_{1}$ and $b_{2}$ are antipodal points on the surface of $B\left(0, r_{0}+d_{0} / 2\right)$ such that $l_{b_{1} b_{2}}$ is parallel to $l_{x_{1} x_{2}}$. After passing $b_{i}$, the curve $C_{i}$ continues on the line in the hyperplane perpendicular to $l_{b_{1} b_{2}}$ that hits $B_{1 / 2}$ in a point with minimal distance to $c_{i}$. 

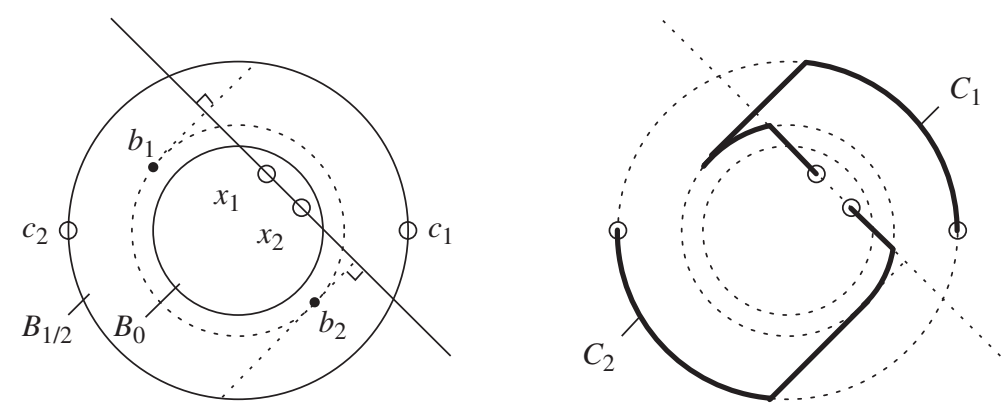

FIgure 3: Construction of the curves $C_{1}$ and $C_{2}$.

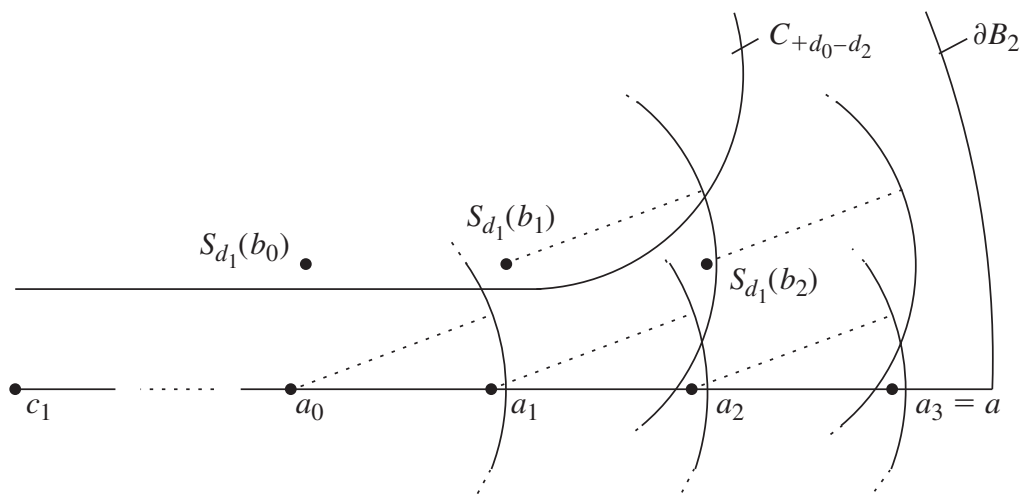

FIGURE 4: Definition of the infection evolution of step $M_{2 \rightarrow 3}^{\prime}$ in the difficult case. Some infection outbursts are indicated by segments of their boundaries, which are connected to their centers by dotted lines.

$M_{1 \rightarrow 2}^{\prime}$ : infecting most of $B_{2}-B_{1}$ with type 2 . We use Lemma 1 to let type 2 grow along $C^{\prime}:=C \cup\left[-r_{1}, c_{2}\right]$ in the time interval $\left(T_{1}^{\prime}, T_{2}^{\prime}\right]$ with precision $\delta:=d_{2} / 2$. This infection evolution defines an event $M_{1 \rightarrow 2}^{\prime}$ with the desired properties.

$M_{2 \rightarrow 3}^{\prime}$ : infecting $D^{1}$ with type 1 . In this step we basically want to let type 1 grow along $\left[c_{1}, r_{2}\right]$ with precision $\delta:=d_{2} / 2$ until it reaches $D^{1}$. In order to keep the 1 -infections close to $\left[c_{1}, r_{2}\right]$, we have to ensure that, for every outburst of type 1 , all points of the corresponding ball that are too far away from $\left[c_{1}, r_{2}\right]$ are already 2 -infected before the outburst occurs. In this case we say that species 2 provides sufficient containment for the outburst. In the following things get more complicated if $D^{1}$ is near $\partial B_{2}$, and, thus, outbursts infecting $D^{1}$ do not yet have sufficient containment. In this case we only let type 1 grow along $\left[c_{1}, r_{2}\right]$ as long as there is sufficient containment. After that we alternatingly produce outbursts of type 2 (providing further containment and possibly infecting part of $\left[c_{1}, r_{2}\right]$ ) and outbursts of type 1 (infecting points close to $\left[c_{1}, r_{2}\right]$ further up); see Figure 4 . It turns out that we have to repeat this scheme at most twice. In the remainder of this subsection we will give a more detailed description of this construction.

For a line segment $l^{\prime}$ of the line $l=l_{c_{1} c_{2}}$, let $S_{r}\left(l^{\prime}\right)$ denote the cylindrical shell with axis $l^{\prime}$ and radius $r$, i.e. the set of all points $x$ with $d(x, l)=r$ such that the projection of $x$ onto $l$ is on $l^{\prime}$. In case of a single point $x$ we write $S_{r}(x):=S_{r}(\{x\})$. In order to determine for which points $a^{\prime} \in\left[c_{1}, a\right]$ an infection outburst at $a^{\prime}$ with precision $\delta$ has sufficient containment on 
$M_{2}^{\prime}$, we first note that

$$
d\left(S_{r}\left(r_{2}-s\right), \partial B_{2}\right) \geq s-r \text { for all } 0 \leq r \leq s \leq r_{2} .
$$

(This and other purely geometric relations will be proved at the end of this subsection.) Equation (14) implies that $S_{d_{0}+2 d_{2}}\left(\left[0, r_{2}-2 d_{0}-d_{1}\right]\right)-B_{1} \subset C$ and, thus,

$$
S_{3 d_{2}}\left(\left[0, r_{2}-2 d_{0}-d_{1}\right]\right) \subset S_{d_{0}+2 d_{2}}\left(\left[0, r_{2}-2 d_{0}-d_{1}\right]\right)_{+d_{0}-d_{2}} \subset C_{+d_{0}-d_{2}} \cup B_{1} .
$$

On $M_{2}^{\prime}$ we know that $C_{+d_{0}-d_{2}}-B_{1}$ is 2-infected, so this shows that the above described infection outburst at $a^{\prime}$ has sufficient containment if $a^{\prime} \in\left[c_{1}, a\right]$ is such that

$$
a^{\prime} \leq r_{2}-2 d_{0}-d_{1}-\left(d_{0}+2 \delta\right)=r_{2}-\left(3 d_{0}+d_{1}+d_{2}\right)
$$

Thus, if $a^{\prime}:=a-\left(d_{0}-2 \delta\right)$ satisfies (16) (i.e. if $\left.a \leq r_{2}-\left(2 d_{0}+d_{1}+2 d_{2}\right)\right)$, we can use Lemma 1 to let type 1 grow along $\left[c_{1}, a^{\prime}\right]$ in the time interval $\left[T_{2}^{\prime}, T_{3}^{\prime}\right]$ with precision $\delta$, and immediately get an event $M_{2 \rightarrow 3}^{\prime}$, as desired. However, if $a>r_{2}-\left(2 d_{0}+d_{1}+2 d_{2}\right)$, we might need two more outbursts (of size $d_{0}$ ) to reach $D^{1}$ with containment not yet provided. We now define $a_{0}<a_{1}<a_{2}<a_{3}:=a$ and $b_{i} \leq a_{i+1}$ to be those points on $\left[c_{1}, a\right]$ satisfying

$$
d\left(a_{i}, a_{i+1}\right)=d_{0}-2 d_{2} \quad \text { and } \quad d\left(S_{d_{1}}\left(b_{i}\right), a_{i+1}\right)=d_{0}+2 d_{2} .
$$

We note that

$$
a_{i} \leq b_{i} \leq a_{i}+\frac{1}{2} d_{1} \quad \text { and } \quad a_{3}=a \leq r_{2}-2 d_{1} .
$$

Figure 4 shows a half-plane starting from $l_{c_{1} a}$. The three points above $\left[c_{1}, a\right]$ indicate the points of intersection of the half-plane with the cylindrical shells $S_{d_{1}}\left(b_{i}\right)$. We define $M_{2 \rightarrow 3}^{\prime}$ to be the following infection evolution (where $T_{2}^{\prime}=t_{0}^{\prime}<t_{1}^{\prime}<\cdots<t_{5}^{\prime}=T_{3}^{\prime}$ ):

1. growth of type 1 along $\left[c_{1}, a_{0}\right]$ in $\left[t_{0}^{\prime}, t_{1}^{\prime}\right]$,

2. growth of type 2 along $S_{d_{1}}\left(\left[b_{0}, b_{1}\right]\right)$ in $\left[t_{1}^{\prime}, t_{2}^{\prime}\right]$,

3. growth of type 1 by a single outburst at $a_{1}$ in $\left[t_{2}^{\prime}, t_{3}^{\prime}\right]$,

4. growth of type 2 along $S_{d_{1}}\left(\left[b_{1}, b_{2}\right]\right)$ in $\left[t_{3}^{\prime}, t_{4}^{\prime}\right]$,

5. growth of type 1 by a single outburst at $a_{2}$ in $\left[t_{4}^{\prime}, t_{5}^{\prime}\right]$.

In every step the growth is meant to be within $B_{2}$ and with precision $\delta$. The important part of the boundary of the infection outburst in every step is indicated in Figure 4. By Lemma 1 we have $\mathrm{P}\left(M_{2 \rightarrow 3}^{\prime}\right)>0$, and Figure 4 shows that, for $M_{2}^{\prime} \cap M_{2 \rightarrow 3}^{\prime} \subset M_{3}^{\prime}$, we only have to ensure the following properties in the corresponding steps. In step 1 we need sufficient containment, i.e. $a_{0}$ has to satisfy (16). This is a consequence of (17), (18), and $8 d_{2} \leq d_{1}$. Also, $B\left(a_{1}, d_{2}\right)$ has to be fully infected, which follows from (17). For step 2, we observe that $b_{0} \leq r_{2}-2 d_{0}-d_{1}$, which follows from (17) and (18). Thus, (15) implies that $S_{d_{1}}\left(b_{0}\right)_{+d_{2}} \subset C_{+d_{0}-d_{2}}$, which means that the growth of type 2 can start from $S_{d_{1}}\left(b_{0}\right)$. No part of $B\left(a_{2}, d_{2}\right)$ is 2 -infected by (17). Also, step 2 has to provide sufficient containment for the next step, i.e. we need

$$
B\left(a_{i}, d_{0}+2 d_{2}\right) \subset\left[0, r_{2}\right]_{+d_{1}} \cup S_{d_{1}}\left(\left[b_{i-1}, b_{i}\right]\right)_{+\left(d_{0}-d_{2}\right)}
$$

for $i=1$. In step $3 B\left(a_{2}, d_{2}\right)$ has to be fully infected, which follows from the first part of (17). Steps 4 and 5 have properties analogous to those of steps 2 and 3, provided that (19) is also 

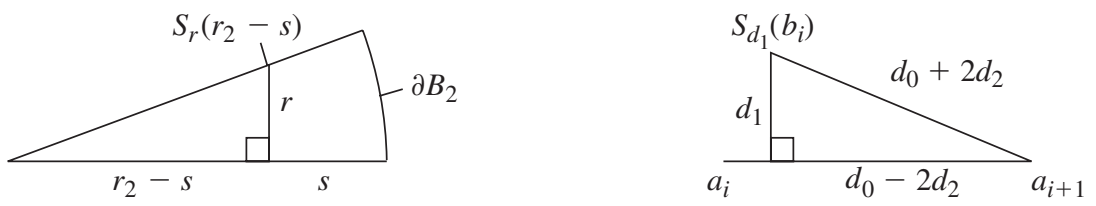

FIGURE 5: Illustrations for the proofs of (14) (left) and (18) (right).

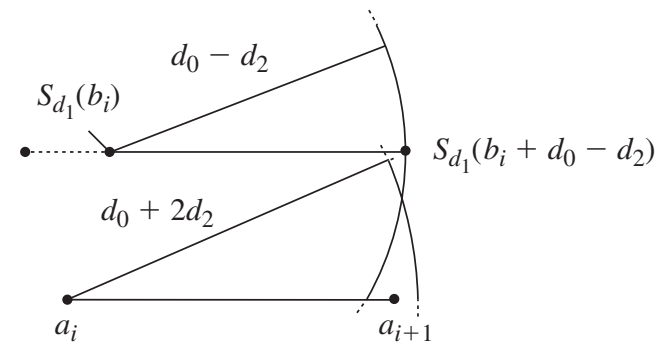

FIGURE 6: Illustration for the proof of (19).

satisfied for $i=2$. Finally, to ensure that the growth is always within $B_{2}$, we need

$$
S_{d_{1}}\left(\left[b_{0}, b_{2}\right]\right)_{+\left(d_{0}+d_{2}\right)} \cup B\left(a_{2}, d_{0}+2 d_{2}\right) \subset B_{2} .
$$

What remains to be checked are properties (14), (18), (19), and (20). As can be seen from Figure 5, assertion (14) is equivalent to

$$
\sqrt{\left(r_{2}-s\right)^{2}+r^{2}}+(s-r) \leq r_{2},
$$

which holds for all $r \leq s \leq r_{2}$. Figure 5 also shows that the first part of (18) is equivalent to

$$
0 \leq d_{0}-2 d_{2}-\sqrt{\left(d_{0}+2 d_{2}\right)^{2}-d_{1}^{2}} \leq \frac{1}{2} d_{1},
$$

which is satisfied for $d_{2} \leq d_{1}^{2} /\left(8 d_{0}\right)$ and $2 d_{1} \leq d_{0}$. The second part of (18) follows by the definitions of $a$ and $d_{1}$. For (19), we observe that $\left[a_{i}-\left(b_{i}-a_{i}\right), a_{i}+\left(b_{i}-a_{i}\right)\right] \subset\left[b_{i-1}, b_{i}\right]$, which follows from (18), so, by symmetry, it suffices to check that

$$
d\left(S_{d_{1}}\left(b_{i}+d_{0}-d_{2}\right), a_{i}\right) \geq d\left(S_{d_{1}}\left(b_{i}\right), a_{i+1}\right)=d_{0}+2 d_{2} ;
$$

see Figure 6. Here the equality follows from (17) and Figure 6 shows that the inequality follows from $a_{i} \leq b_{i}$ and $b_{i}+d_{0}-d_{2} \geq a_{i}+d_{0}-d_{2}>a_{i+1}$. For (20), we first observe that $B\left(a_{2}, d_{0}+2 d_{2}\right) \subset B_{2}$ follows from $r_{2}-a_{2} \geq 2 d_{1}+\left(d_{0}-2 d_{2}\right) \geq d_{0}+2 d_{2}$, where we have used (18). Furthermore, we have

$$
S_{d_{1}+d_{2}}\left(r_{2}-d_{0}-d_{1}-2 d_{2}\right)_{+d_{0}+d_{2}} \subset B_{2},
$$

by (14), so it suffices to show that $b_{2} \leq r_{2}-d_{0}-d_{1}-2 d_{2}$. This follows from (17) and (18).

$M_{3 \rightarrow 4}^{\prime}$ : infecting the remaining part of $B_{2}-B_{1}$ with type 2 . Let $r_{2}^{\prime}:=d\left(S_{d_{1}+d_{2}}\left(r_{2}-d_{0}-\right.\right.$ $\left.\left.d_{1}-2 d_{2}\right), 0\right)$ and $C^{\prime}:=B\left(0, r_{2}^{\prime}\right)-B_{1}-\left[0, r_{2}\right]_{+d_{1}+d_{2}}$. We define $M_{3 \rightarrow 4}^{\prime}$ by the growth of type 2 along $C^{\prime}$ in the time interval $\left[T_{3}^{\prime}, T_{4}^{\prime}\right]$ with precision $\delta:=d_{2} / 2$. By Lemma 1 we have $\mathrm{P}\left(M_{3 \rightarrow 4}^{\prime}\right)>0$ and, for $M_{3}^{\prime} \cap M_{3 \rightarrow 4}^{\prime} \subset M_{4}^{\prime}$, it suffices to observe that on $M_{3}^{\prime}$ no point of $C_{+d_{2}}^{\prime}$ is 1-infected, most of $C^{\prime}$ is 2-infected, by (14) we have $C_{+d_{0}+d_{2}}^{\prime} \subset B_{2}$, and, by the choice of $r_{2}^{\prime}$ we have $C_{+d_{0}-d_{2}}^{\prime} \supset C_{+}$. 


\subsection{Restricting the strong type: Lemma 7}

In this subsection we restrict our attention to the infection evolutions of $Z:=Z(\Gamma)$ and $\tilde{Z}$ for a fixed point configuration $X \in M_{\mathrm{in}, \mathrm{fi}}$. By the definition of $M_{\mathrm{in}, \mathrm{fi}}$ we know that $Z^{2}$ grows without bound and that $X$ contains an infinite type- 1 infection path $\left(p_{n}\right)_{n \geq 1}$ with respect to $Z$ such that, for $p_{1}=\left(x_{1}, t_{1}, r_{1}, b_{1}\right)$, we have $x_{1} \in D^{1}$ and $t_{1} \geq T\left(D^{1}\right)$. It suffices to show that

- $\tilde{Z}_{t}^{2} \cap B_{2}^{c} \supset Z_{t}^{2} \cap B_{2}^{c}$ for all $t \geq 0$, and

- $\left(p_{n}\right)_{n}$ is an infinite type- 1 infection path with respect to $\tilde{Z}$.

To prove the first assertion, we show that, for all $t \geq 0$,

$$
\tilde{Z}_{t}^{\cup} \subset Z_{t}^{\cup}, \quad \tilde{Z}_{t}^{1} \subset Z_{t}^{1}, \quad \text { and } \quad Z_{t}^{2} \cap B_{2}^{\mathrm{c}} \subset \tilde{Z}_{t}^{2} \cap B_{2}^{\mathrm{c}}
$$

by induction on the times of growth of both models. At $t=0$ the above is trivial, so suppose that $t>0$ is a time of growth. If the growth is due to the initial configuration of $\tilde{Z}$, the inclusions remain true, because in the model $Z$ every fundamental ball is infected at its time horizon. Otherwise, the growth is due to an outburst $p=(x, t, r, w) \in X$ in one of the models. If $p$ produces an effective type- 1 outburst in $\tilde{Z}$, the same outburst happens in $Z$. If $p$ produces an effective type- 2 outburst in $\tilde{Z}$, there is also an outburst of type 1 or type 2 in $Z$. So if $p$ produces an effective outburst with respect to $\tilde{Z}$, all inclusions remain true. Now we take a look at the remaining case, i.e. $p$ produces an effective outburst in $Z$ and not in $\tilde{Z}$. We only have to worry about the third inclusion, and, thus, we only have to consider the case that $p$ produces an effective type-2 outburst in $Z$ such that $B(x, r) \not \subset B_{2}$ and $x \in B_{2}$. (If $B(x, r) \subset B_{2}$, the outburst contributes to neither side, and if $x \notin B_{2}$, the outburst contributes to both sides.) In this case $x$ is in one of the fundamental balls and $t$ is after the corresponding time horizon. Thus, the initial configuration of $\tilde{Z}$ ensures that the same outburst happens in $\tilde{Z}$ and the third inclusion remains true.

For the second assertion, we show by induction that $p_{n} 1$-infects $x_{n+1}$ in $\tilde{Z}$. By the induction hypothesis, $x_{n}$ gets 1 -infected in both models at time $t_{n}$, so we have a type-1 outburst at time $t_{n+1}$ in both models. Since $\tilde{Z}_{t}^{\cup} \subset Z_{t}^{\cup}$ at all times, $x_{n+1}$ cannot get infected in $\tilde{Z}$ earlier than in $Z$. Thus, the outburst 1-infects $x_{n+1}$ in $\tilde{Z}$ as well.

\section{Acknowledgement}

We would like to thank H.-O. Georgii for bringing this topic to our attention and for helpful discussions and remarks.

\section{References}

[1] Deijfen, M. (2003). Asymptotic shape in a continuum growth model. Adv. Appl. Prob. 35, 303-318.

[2] Deijfen, M. and Häggström, O. (2004). Coexistence in a two-type continuum growth model. Adv. Appl. Prob. 36, 973-980.

[3] Deijfen, M. and Häggström, O. (2006). The initial configuration is irrelevant for the possibility of mutual unbounded growth in the two-type Richardson model. Combinatorics Prob. Comput. 15, 345-353.

[4] Deijfen, M., Häggström, O. And Bagley, J. (2004). A stochastic model for competing growth on $\mathbb{R}^{d}$. Markov Process. Relat. Fields 10, 217-248.

[5] Häggström, O. and Pemantle, R. (1998). First passage percolation and a model for competing spatial growth. J. Appl. Prob. 35, 683-692.

[6] Richardson, D. (1973). Random growth in a tessellation. Proc. Camb. Phil. Soc. 74, 515-528. 